\title{
Soft Office: a human-robot collaborative system for adaptive spatial configuration
}

\author{
Maria Yablonina ${ }^{1,2} \odot \cdot$ Brian Ringley $^{3} \cdot$ Giulio Brugnaro $^{4} \cdot$ Achim Menges $^{1}$
}

Received: 15 June 2020 / Accepted: 3 February 2021 / Published online: 10 March 2021

(c) The Author(s), under exclusive licence to Springer Nature Switzerland AG part of Springer Nature 2021

\begin{abstract}
The Soft Office project was developed in response to the rapidly changing context of commercial architecture, where accommodating fluid programmatic requirements of occupants has become key to sustainable interior space. The project is placed within a broader context of relevant research in architectural robotics, in situ robotic fabrication, and adaptive and reconfigurable architecture. It establishes a methodology for spatial configuration through the implementation of a custom collaborative robotic interior reconfiguration system. Within this system, human users and task-specific robots perform complementary tasks toward a dynamic spatial goal that is defined by a set of evaluative criteria intended to predict successful interior space configurations (Bailey et al. in Humanizing digital reality: design modeling symposium Paris 2017, Springer Singapore, Singapore, pp 337-348, 2018). Venturing beyond robotics as merely a means of construction automation, the presented research deploys an approach that critically engages future models of interaction between humans and robotic architecture, mediated by in situ, architecturally embedded machines. In contrast to a conventional collaborative robotic manufacturing process, where a human worker is executing fabrication and manufacturing tasks according to a pre-designed blueprint, the proposed approach engages the human user as the designer, the worker, and the consumer of the architectural outcome. This gives the occupant the agency to rapidly reconfigure their environment in response to changing programmatic needs as well as the ability to respond ad hoc to outside forces, such as social distancing requirements for the post-quarantine reoccupation of buildings. Furthermore, task-specificity of the presented robotic system allows us to speculate on future roles of designers in the development of architectural fabrication technology beyond the appropriation of existing hardware and to look towards systems that are architecture specific.
\end{abstract}

Keywords Task-specific robotics · Single task construction robots $\cdot$ Reconfigurable architecture $\cdot$ In situ fabrication . Commercial architecture $\cdot$ Interior architecture

\section{Introduction}

Maria Yablonina

maria.yablonina@daniels.utoronto.ca

Brian Ringley

bringley@bostondynamics.com

1 Institute for Computational Design and Construction, University of Stuttgart, Keplerstrasse 11, 70174 Stuttgart, Germany

2 Daniels Faculty of Architecture, Landscape, and Design, University of Toronto, 1 Spadina Crescent, Toronto, ON M5S 2J5, Canada

3 Construction Technology, Boston Dynamics, Boston, USA

4 The Bartlett School of Architecture, University College London, Gower Street, London WC1E 6BT, UK
The Soft Office project aims to investigate and develop a robotic, computational, and architectural approach to the design of a reconfigurable interior environment tailored to physically adapt to its occupant's needs. It proposes an in situ architectural-robotic system for interior space configuration (Fig. 1). The research is placed within the discourse of Architectural Robotics (Gross and Green 2012) and Robotic Building (Bier 2018), as well as reconfigurable architecture, and collaborative robotics; proposing to combine manual and automated processes for reconfiguration and adaptation, leveraging both human agility and robotic ability to perform repetitive tasks toward complex spatial solutions in a highly dynamic interior environment. 
Fig. 1 Soft Office robotic system detail

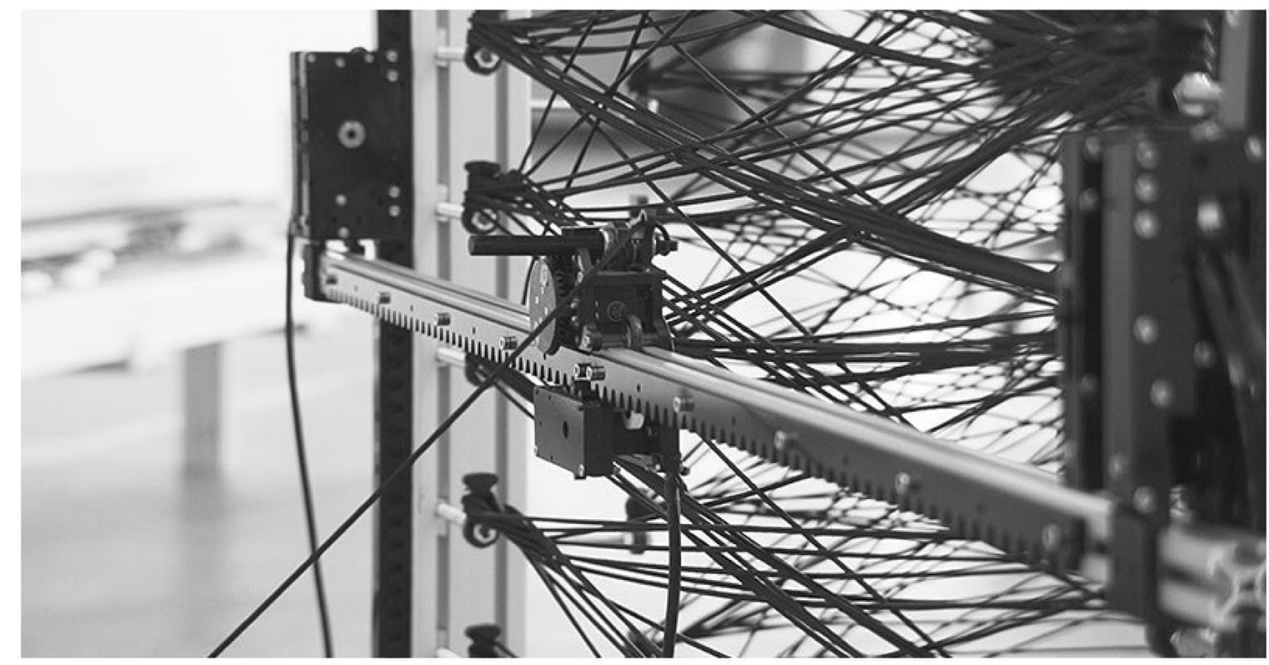

The presented research includes a full-scale prototype of a semi-robotic partition wall, as well as a software system with tools for computational design, user interaction, and robot control. The development of the robotic, architectural, and computational aspects of this work was conducted in an integrative design process, where technical and architectural design parameters were considered simultaneously as interdependent variables. Task-specificity of the custom robotic and software tools and the architectural infrastructure allowed us to develop a system tailored for reconfiguration scenarios in contrast to conventional robotic fabrication approaches in architecture (Bock and Linner 2015, 2016).

The project was developed for, and implemented during, the research workshop hosted by the Smartgeometry 2018 conference at the Daniels Faculty of Architecture, Landscape and Design at the University of Toronto. The workshop format provided a unique opportunity to test and evaluate not only the system itself, but also the designer's experience of interacting with it. Throughout the workshop, the participants acted as inhabitants, artifact designers, and digital workflow developers, generating insight into all aspects of the research.

\section{Background}

\subsection{History of reconfigurable architecture}

Topics of reconfiguration, adaptation, and interactivity have been present in the architectural discourse as early as Le Corbusier's Maison Dom-Ino, creating "a juxtaposable system of construction according to an infinite number of combinations of plans" (Turner 1987). These ideas of flexible and reconfigurable architectural systems became radicalized in the 1960s when Archigram first proposed their speculative vision of the future city as mechanized "living equipment," arguing against outmoded practices of fixed walls and spaces in favor of continuous cultural and environmental rearrangements (Sadler 2005). In the 1970s, The Architecture Machine group envisioned "a man-made environment that responds to and is meaningful for him or her" (Negroponte 1975) and Cedric Price introduced the Generator Project, which denoted the non-human agency in the built environment (Steenson 2017).

In the late 1990s and early 2000s, technological developments in the fields of mechanics, actuators, and sensors provided an opportunity to explore beyond speculation, establishing a field of interactive architecture shaped by the "...contemporary discourse focusing on interaction in human technology interfaces..." (Kilian 2018). Most early interactive architecture projects were focused primarily on two-dimensional and two-and-a-half dimensional architectural elements, like façades and walls, rather than space configuration and its programmatic function. Today, in the context of rapid urbanization, the conversation is inevitably shifting away from screen-like applications towards continuous adaptation of architectural space to its inhabitants needs, rendering it as "robots for living in" (Mitchell 1999).

\subsection{State of the art}

In the field of architectural research, the topic of reconfigurable and adaptive architecture has been addressed through a variety of methods and approaches, ranging from manually actuated hybrid material enclosures (Sparrman et al. 2017) and fully reusable granular materials that can be rearranged into any shape (Dierichs and Menges 2017) to room-size robots (Kilian 2018) and modular systems reconfigured by aerial vehicles (Wood et al. 2019). In the pursuit of interactive spaces, the discourse around robots in architecture has expanded beyond machines for production and fabrication towards Robotic Building (Bier 2018) and Architectural 
Robotics (Green 2016). This implies the necessity of design "not only at production level, but also at building operation level, wherein users and environmental conditions contribute to the emergence of various architectural configurations" (Bier 2018). A series of projects executed by the Hyperbody group at the University of Delft closely examined the relationship between Robotic Production and Robotic Operation (Cheng 2016), arguing that building components are cyberphysical and must be informed by structural, environmental, assembly, and operation considerations. The Architectural Robotics lab at Cornell University has developed a variety of architectural objects informed by human needs (Schafer et al. 2018). The Animated Work Environment project focused specifically on the development of a robotic system that can change its shape in response to a variety of userdefined programs in a workspace (Houayek et al. 2014).

In industry, there is a growing interest in roboticized and manually reconfigurable spaces as can be traced by the number of recent start-ups focusing on the topic (e.g., Asmbld, Kasita, Blokable). This interest is particularly acute within the context of commercial interior program such as workplace and retail architecture, as the intensive informational nature of a business and its need to continually adapt makes the investment in technical development more economically viable compared to other areas of the built environment (Kelly 1994). The under-occupied and under-used Taylorist office building can no longer support emerging ways of working due to its inflexibility (Duffy 2008) and requires rethinking. Thus, this project proposes an architecturally embedded robotic system for in situ construction and reconfiguration of interior space.

The implementation of robotic equipment in the context of an occupied architectural environment requires addressing the issues of scale of the robotic work envelope in relation to the safety and practicality of its operation. Conventional robotic equipment used in fabrication and manufacturing requires implementation of safety protocols that restrict human access to robot operation areas. Alternatively, developments in the field of collaborative robotics demonstrate novel hardware systems augmented with real-time sensing capabilities that make them safer (KUKA Roboter $\mathrm{GmbH} 2015$ ), thus offering collaborative workflows where machines and human workers work alongside each other (Stumm et al. 2019). However, the work envelopes of collaborative robots tend to be significantly smaller than those offered by conventional industrial equipment.

To address this relationship between scale and safety, Soft Office proposes the implementation of small, low-payload robots in combination with soft materials (Yablonina and Menges 2019) and human-robot workflows to actualize work envelopes of architectural scale enabled by human-robot cooperation and strategic task distribution. Specifically, the project proposes an architecturally embedded robot, conceptually positioned at the intersection of mobile robotics and architectural robotics. Treating the robotic system as a plug-in to an architectural space avoids conventional problems of mobile robotics such as mobility, localization, position repeatability, and self-charging in dynamic environments, while accommodating flexibility and deployability in situ at a variety of sites without the cost, operational overhead, and structural requirements of robotic architectural elements.

\section{Methods}

The Soft Office system consists of a modular architectural infrastructure, a deployable robotic fabricator, and a software system with tools for computational design, user interaction, and robot control. The architectural infrastructure, designed to be reconfigurable by human users, consists of rigid linear vertical elements that can be placed anywhere in a rectangular grid to approximate a typical commercial architecture interior structure. The vertical elements serve as a substrate for the deployable robotic system that leverages them as a boundary for its work envelope. The robotic system produces partition surfaces by winding filament material between the vertical elements (Fig. 2). The use of an additive material technique such as filament winding allows for a variety of material layers and configurations to economically simulate architectural properties of transparency, privacy, insulation, and acoustics. The inherent reversibility of filament wound surfaces provides opportunities for an adaptive architectural element that is regenerative, decoupling continuous demolition and construction activity from the consumption of finite material resources. Furthermore, the lightweight filament material allows for the deployment of a low-payload robotic system that can safely co-occupy a habitable space with small, quiet, and energy efficient motors. The computational design tool allows the users to explore the design space of the architectural-robotic system, preview configurations in the digital space, and generate the robotic routine commands and human operator instructions for the execution of the selected spatial configuration. The user interaction interface allows the user to monitor the overall system and keep track of changes made in the physical space.

The system enables two approaches to architectural change: manual global reconfiguration of primary architectural elements in the space, and local robotic adaptation of the wound surfaces. This task distribution amongst human and non-human actors ensures systematic efficiency of the overall system by allowing the human to quickly accomplish a high-payload dexterous task that would be challenging to automate, while assigning a continuous and repetitive task to a machine. Beyond achieving a cost- and time-efficient system, these two approaches enable a combination of two 


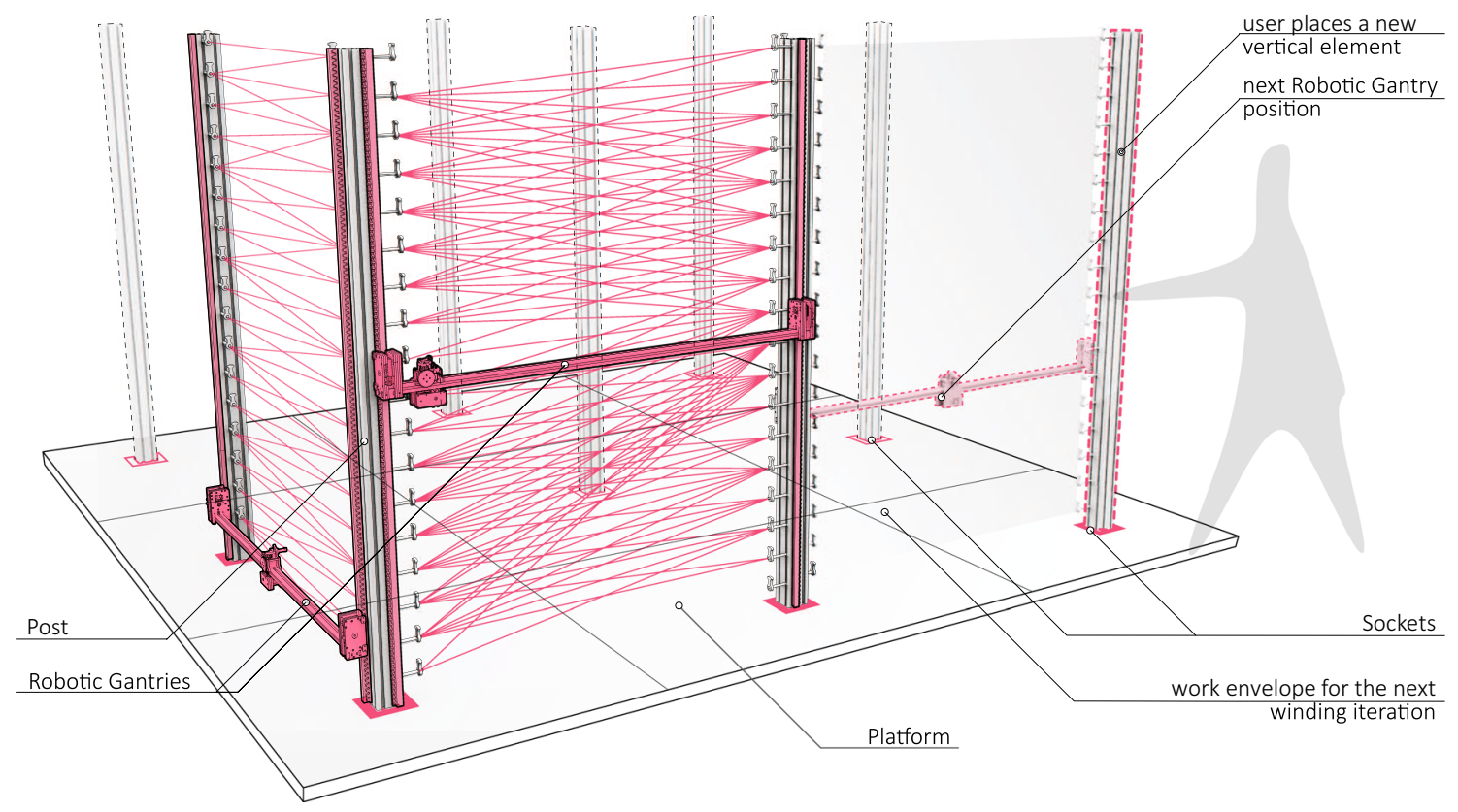

Fig. 2 Overall architectural system diagram

temporal modes of change: immediate and continuous. The immediate mode allows a user to change the spatial configuration of the workplace to accommodate new programs (e.g., individual work, teamwork, meeting, etc.), while the continuous mode offers a perpetual response to gradually changing workplace criteria like natural lighting, privacy, and sociability via tailored transparency of the wound partitions.

\subsection{Architectural system}

The architectural infrastructure of the Soft Office system consists of two parts: Platform and Post, augmented with a Robotic Gantry (Fig. 2). The Platform is equipped with Sockets: networked elements enabled with sensors, designed to hold a Post and recognize if it has been placed. The Post is a primary structural element in the system and is equipped with custom hardware for the Robotic Gantry locomotion along its length as well as an array of anchors about which the partitions are wound. The Posts can be moved and installed into any Socket in the Platform, and the Robotic Gantry can be deployed between any two neighboring Posts.

The user is free to move the Posts around to establish a variety of architectural conditions based on spatial intuition. Once the desired configuration is finalized, the user can place the Robotic Gantry at the required locations in order to begin the winding process. Alternatively, a top-down design approach to spatial configuration is possible, where the user can configure the desired space digitally and place the Posts and Robotic Gantries following the resulting model. Potentially, a fully intuitive interaction mode could be achieved where the user interacts with the system entirely through Post and Gantry placement, relying on the robotic counterpart to intelligently respond, eliminating any screen-based interaction.

\subsection{Robotic system}

The Robotic Gantry is a two-degrees-of-freedom custom machine capable of travel and anchoring routines that enable the winding fabrication of the partition surfaces (Fig. 3). It consists of three robotic modules that leverage the Posts of the architectural system as a locomotion substrate. This substrate can be accommodated in either negative space, such as a reveal or channel within an extruded aluminum post, or in positive space such as a strip of furring attached to an existing cast-in-place concrete column (Yablonina et al. 2020). The vertical travel of the Robotic Gantry is enabled by two identical vertical Locomotion Modules, each consisting of an actuator and a custom rail clamping assembly. A pair of Locomotion Modules carries a horizontal rail equipped with a Winding Module that can travel along it. The Winding Module consists of a locomotion assembly and a winding effector mechanism: a linearly actuated arm with an eyelet that is guiding the filament loop. Both vertical and horizontal robotic modules rely on a rack and pinion method for travel, augmented with an internal optical encoder, which provides accurate positioning without the need for localization.

The fabrication process consists of two robotic routines: travel and winding. The travel routine actuates both Locomotion Modules to move to the desired location. The 


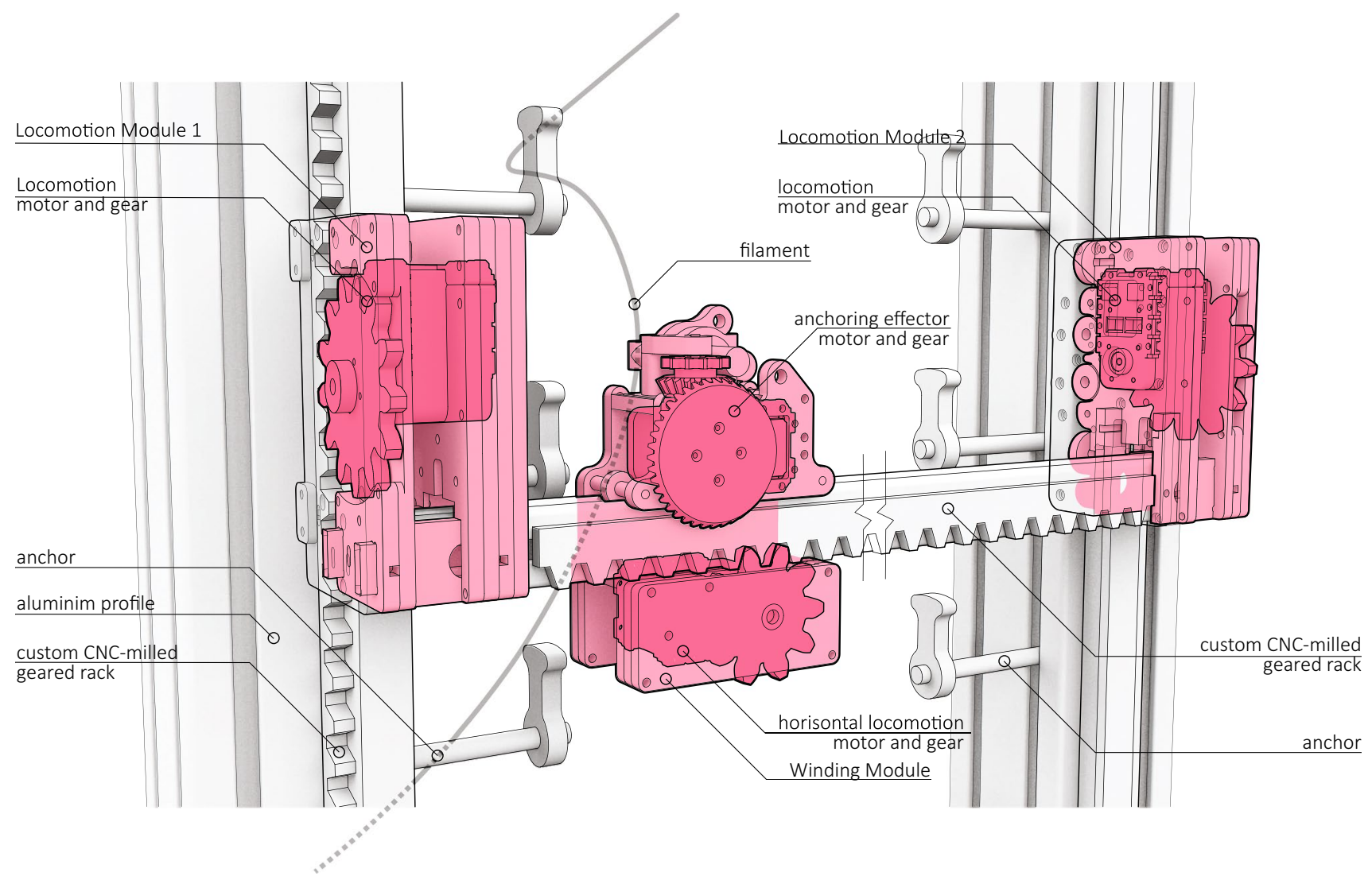

Fig. 3 Robotic gantry system overview

winding routine consists of sequential series of movements performed by all three robotic modules as follows: (1) the Locomotion Modules position the horizontal rail $1 \mathrm{~cm}$ above the anchor at the programmed winding location, (2) the winding arm is extended, placing the eyelet behind the anchor cap, (3) the Locomotion Modules bring the rail down to the anchor height, (4) the winding arm is reeled back, guiding the filament to loop around the anchor cap, and (5) the Robotic Gantry proceeds towards the next winding location, leaving a loop of filament wrapped around the anchor. Sequential execution of the winding and traveling routines allows the Robotic Gantry to wind filament surfaces between two Posts according to the designed winding syntax.

Deployment of the Robotic Gantry on a pair of Posts requires a preliminary calibration routine executed by the user. Once the gantry is installed, the user manually pushes the gantry down to the ground, and the winding module to a defined location marked on the horizontal rail, establishing a relative origin for the coordinate space of the robotic process within the plane of the wound partition. All robotic modules act as individual clients of a central server that issues fabrication routine commands in accordance with the input provided by the computational design tool. In the workshop, the commands were issued to the robot clients from a direct USB connection to the server computer, but the robot hardware and system network configuration could be easily modified to allow a local machine or web host to control the robot clients over a network.

\subsection{Computational design tool}

The computational design tool developed for the project includes user interaction features operating at the global and local levels of the architectural system (Fig. 4). The global level addresses Post placement in the given space and resulting Partition configurations. The local level addresses the winding syntax pattern within the constraints of the robotic system's limitations. The proposed interface allows for the communication between high-level design intentions and low-level robot control operations. This integrated workflow aims to seamlessly support the reconfigurability of the system through multiple winding sessions, effectively blurring the boundaries between the stages of design and robotic operation and allowing the user to directly inform the qualities of the space without the necessary mediation of a third party (e.g., manufacturer, on-site contractor, programmer, etc.). 


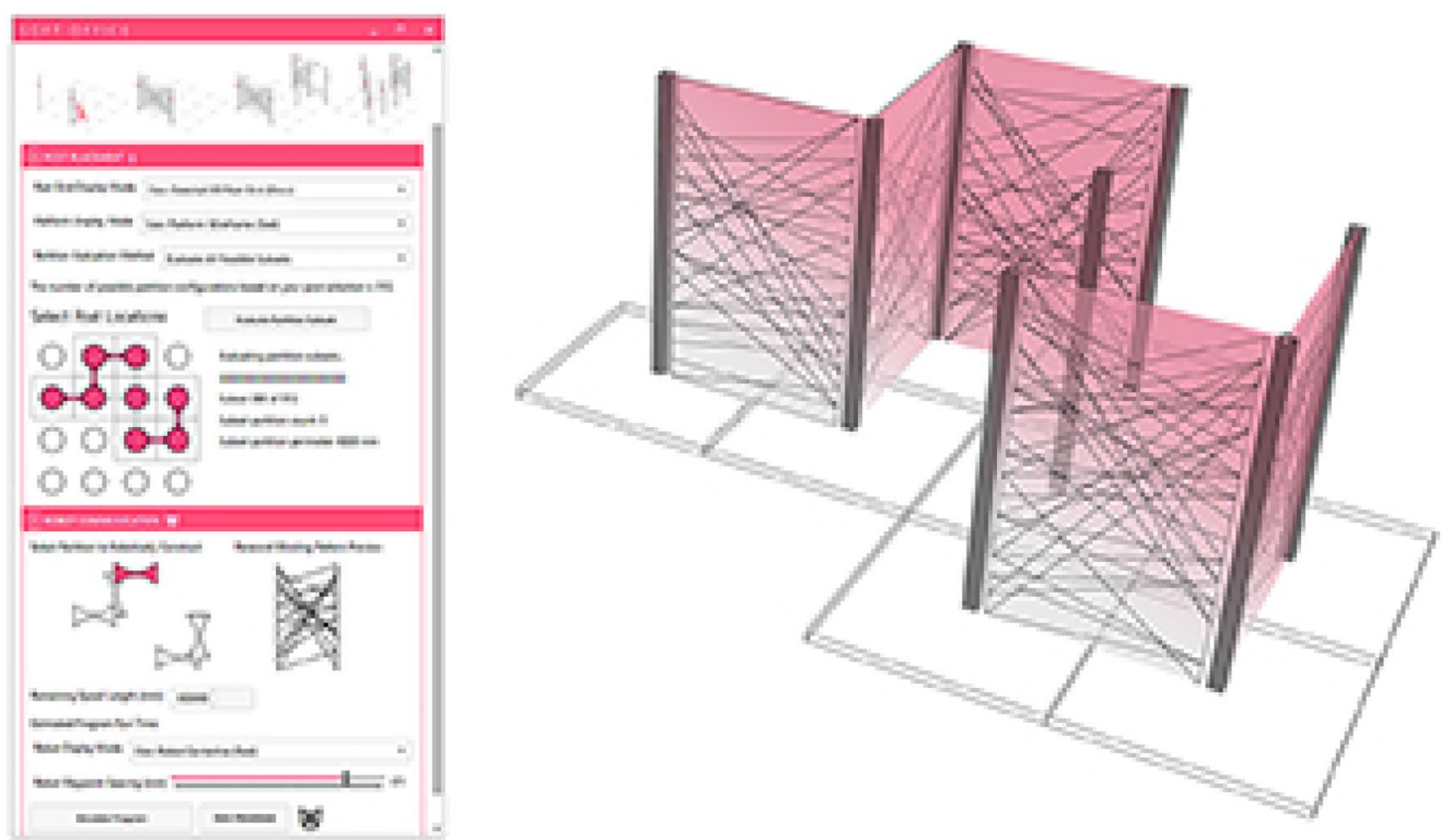

Fig. 4 Computational design tool: partition configuration preview and UI (screengrab)

The interface acts as a digital preview, presenting the vast design space generated by the computational tool to the user, and suggesting the available enclosure options for a given Post configuration. It provides the user with control over the partition design parameters and ensuing robotic winding instructions and supports and reinforces the user's intuition with real-time feedback. In addition to presenting the combinatorial logic of options, the interface assists the user in their selection of a design output by providing quantitative partition data. This includes configuration data, such as partition count, as well as fabrication constraints such as maximum amount of filament per anchor and overall length of material used. Moreover, the system provides a range of spatial metrics such as aspect ratio, solidity, and open space which can be utilized in combination to evaluate the success of a given layout (Bailey et al. 2018). This accessible and interactive interface paradigm is made possible by the Human UI library which allows for Windows Presentation Foundation (WPF) interface design and Extensible Application Markup Language (XAML) styling in the Grasshopper node-based visual programming environment.

At the global scale, the user begins the design process by either inheriting an interface visualization of the sensed physical Post placements, or by explicitly selecting desired Post locations in the digital design space. Once the Post is placed or selected, the computational model is updated to include the new position of the added element. Every time a Post is moved or introduced into the system, the computational design tool suggests a variety of partition configurations for the user to choose from based on the parameters of the space as well as the robotic and structural limitations of the system. Once computed, the design space catalog can be explored manually with an interface slider or automatically animated, depending on which interface option the user selects. The solutions are simultaneously drawn as a two-dimensional plan view and rendered as an orbitable three-dimensional view in the display window. To assist the user in the selection of a Partition arrangement, each configuration within the design space reports a set of values based on the selection criteria defined by the user. If desired, the user may override computed solutions by explicitly selecting the desired Partitions in the interface, which will automatically restrict selection to grid edges with a placed Post on both ends. Once a given Partition solution is selected for fabrication, the Partition elevations are drawn into the interface where they serve to visualize the winding patterns to be computed based on an ensuing set of user input specific to the local wound partition design.

At the local scale, the computational model is used for generating the winding syntax, which consists of a series of winding behaviors informed by the user-defined criteria, such as the modulation of the filament density in the partition (Fig. 5). The user can locally control the desired density using a scalar field to assign dynamic scores to each anchor and preview it digitally through a grayscale gradient map. Furthermore, the system includes the opportunity to specify fabrication and material constraints such as the length of the thread, the maximum number of operations, the winding 

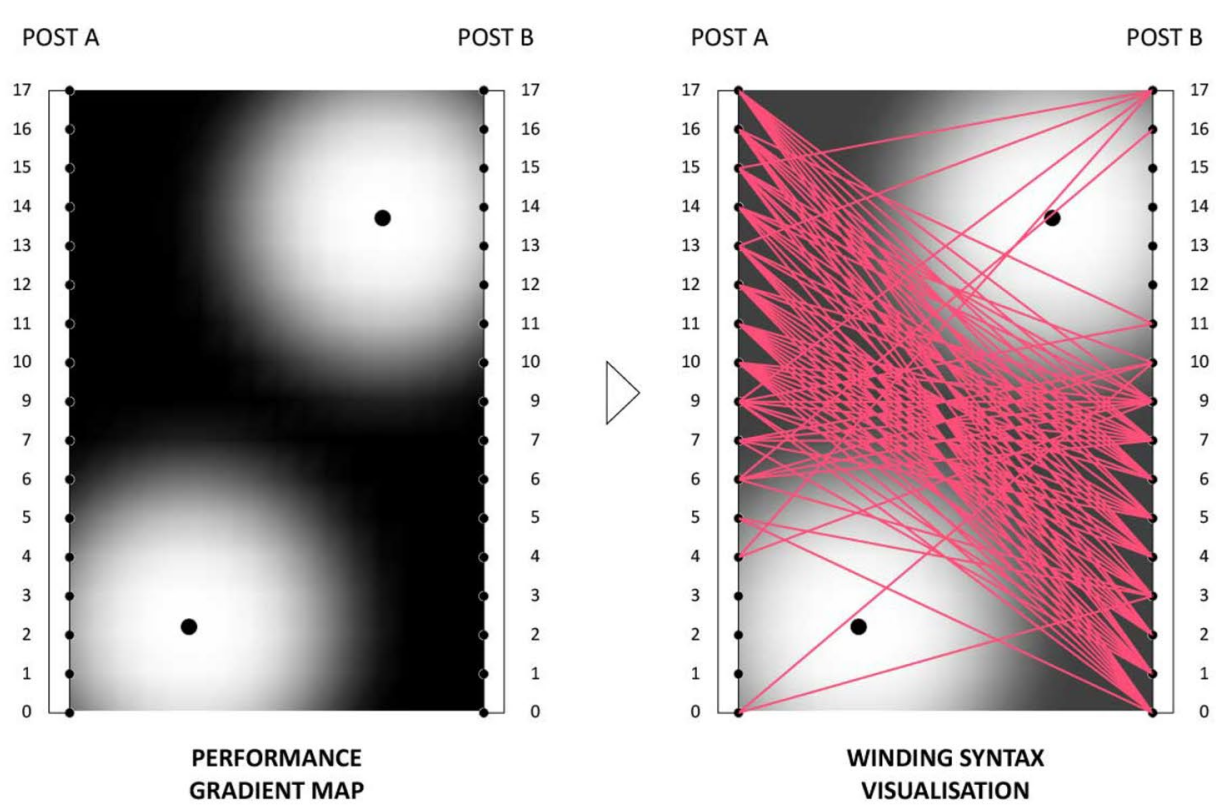

$\begin{array}{ccc}\begin{array}{c}\text { Anchor } \\ \text { Index }\end{array} & \begin{array}{c}\text { Motor } \\ \text { Rotations }\end{array} & \begin{array}{c}\text { Distance } \\ {[\mathrm{mm}]}\end{array} \\ \text { A12 } & 4.75 & 787.5 \\ \text { B05 } & 4.07 & 675 \\ \text { A11 } & 7.46 & 1237.5 \\ \text { B00 } & 10.18 & 1687.5 \\ \text { A15 } & 8.82 & 1462.5 \\ \text { B02 } & 4.75 & 787.5 \\ \text { A09 } & 3.39 & 562.5 \\ \text { B04 } & 6.10 & 1012.5 \\ \text { A13 } & 4.07 & 675 \\ \text { B07 } & 2.03 & 337.5 \\ \text { A10 } & 4.75 & 787.5 \\ \text { B03 } & 8.82 & 1462.5 \\ \ldots & \ldots & \ldots\end{array}$

Fig. 5 Computational design tool: winding syntax generation logic

time or the maximum amount of filament that can be carried by one anchor. In the computational model, the physical components of the system have been abstracted into the three main object types of Partition, Post, and Anchor, and their relationship is made explicit in a hierarchical map to efficiently query the state of each element. The advantage of such an approach is to establish a set of rules that will dynamically determine the next point in the winding syntax. These rules are based on an evaluation of the current state of the wound partition constrained by the desired performance and fabrication parameters.

The computational design tool output is a sequence of numerical values for the robotic actuation automatically generated from the winding syntax, translating the relative distance between anchors to the gear motor displacement. Initialized in the interface by the user, these values are directly streamed to the control system of the calibrated Robotic Gantry, while the user maintains control over the winding through a series of commands that allow for continuous intervention and modifications of the design during its production.

\section{Results and reflection}

The outcome of this research was set up and tested in the context of the Smartgeometry 2018 research workshop. The hardware elements and the robotic system were developed and produced prior to the event and installed on-site for the workshop participants to build and experiment with. Participants were invited to use the proposed software system to generate architectural behaviors and winding sequences, as well as to build upon the software and test new features with the physical setup. The outcome of the workshop was a five by five meter demonstrator of the architectural-robotic system, including eight Posts, two Robotic Gantries, and six wound Partitions (Fig. 6).

The robotic system has successfully performed winding routines on three partitions based on the winding syntax design input generated throughout the workshop. Additionally, three partitions were wound manually, providing an opportunity to benchmark the complexity of achievable geometry according to the physical limitations of the robots, as well as establish error rate and labor-hour baselines for the communication of the winding syntax and the production of the wound surfaces, respectively. When comparing the speed of manual winding to the speed of robotic winding, a single line of filament wound between two anchors took a couple seconds to be done by a human and approximately seventeen to twenty-five seconds by the robot (depending on the distance between the anchors). However, when benchmarking the manual and the robotic processes at the scale of a full partition it became clear that the manual process is significantly delayed by the instruction sorting process: the anchors had to be labeled and the person performing the winding had to check with the winding syntax sheet at every iteration which resulted in a slow, cumbersome, and error-prone process. A full partition of 8 layers took approximately 45 min to wind robotically and approximately an hour manually.

Additionally, the team has performed unwinding partition tests during the project setup phase. The unwinding 


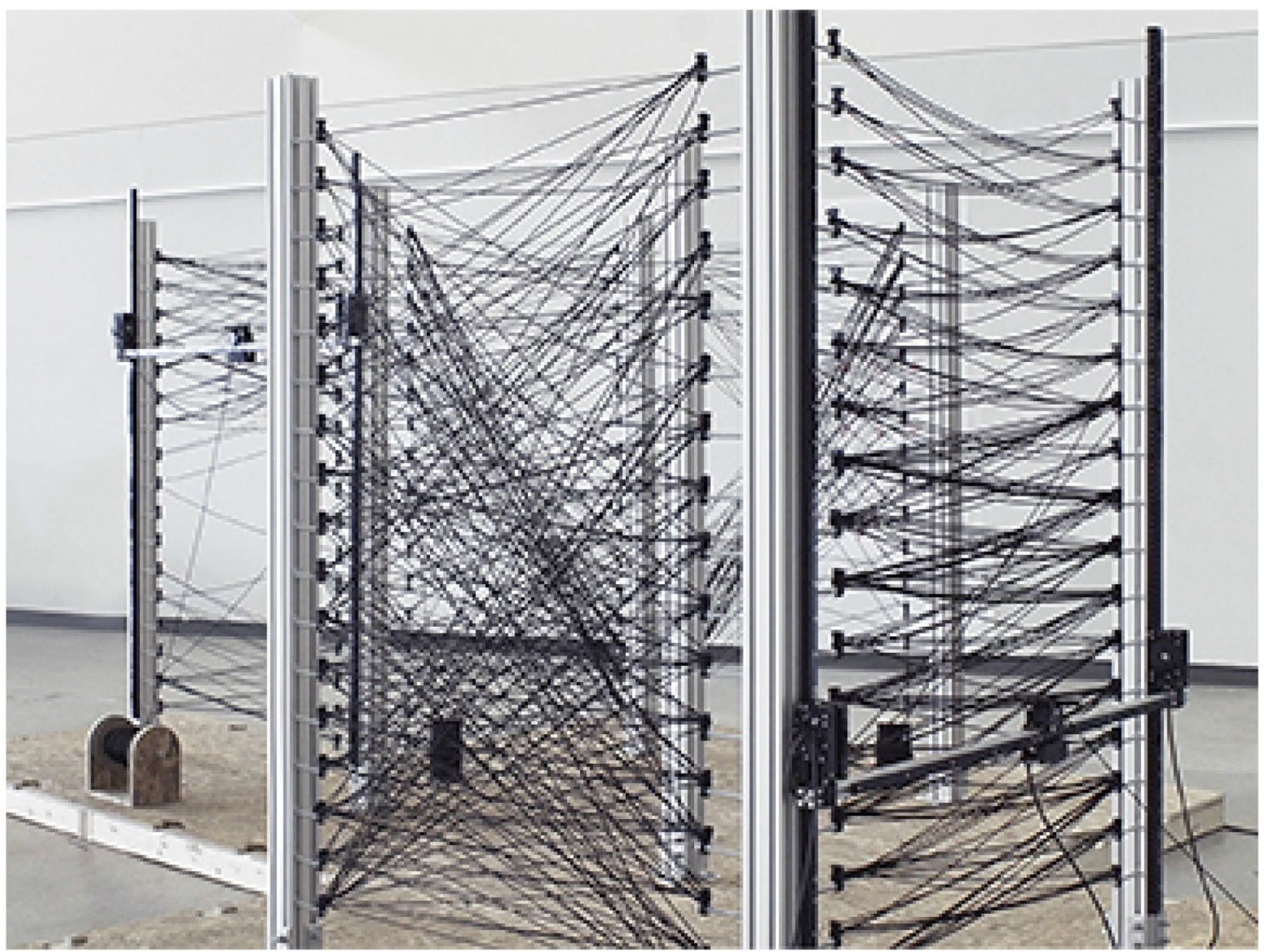

Fig. 6 Demonstrator of the Soft Office system installed during the (blinded) conference

routine was successfully performed by simply running the robotic routines backwards. However, to fully implement the unwinding behavior in an unsupervised context, an actuated filament feeding device is required. In its current state the Robotic Gantry relies on a passive spool holder to supply the material, wherein the filament tension is ensured by mechanical friction, and thus does not allow for material reeling back onto the spool.

Throughout the workshop, the group deployed a variety of methods to design and prototype their ideas, ranging from manual model making to experimenting with the provided computational design tool and the implementation of new software modules (Fig. 7). The initial series of manually wound small-scale models allowed the participants to quickly build material intuition within the system and understand the relationship between the architectural parameters and the inherent properties and limitations of a two-dimensional continuous winding system. This intuition informed the development of winding behaviors in the digital interface and the assessment of the actual design scenarios with the robotic system, considering the full range of fabrication parameters and constraints.

\subsection{Architectural system evaluation and future development}

In the future, issues of acoustics and air flow would have to be addressed in relation to the functional separation of commercial interiors, particularly in office environments. COVID-19 and the need to de-densify interior space for post-quarantine office and retail building re-occupation has further demonstrated the importance of layout reconfiguration, and more critically the building occupant's agency as designer/builder/user to expedite urgently required layout changes to their environment. While this is generally accommodated by the Soft Office project, this should be addressed with more specificity in the future work. A modular rectangular grid of a set dimension provides a straightforward means of implementing regular spacing of seating and desks, but it does not account for many critical interior space utilization factors such as the air movement through the building (Lu et al. 2020) and occupant behavior such as interaction and duration of interaction (Park et al. 2020). There are also opportunities to more directly address the material and density of the partitions, and to integrate metrics such as ease 

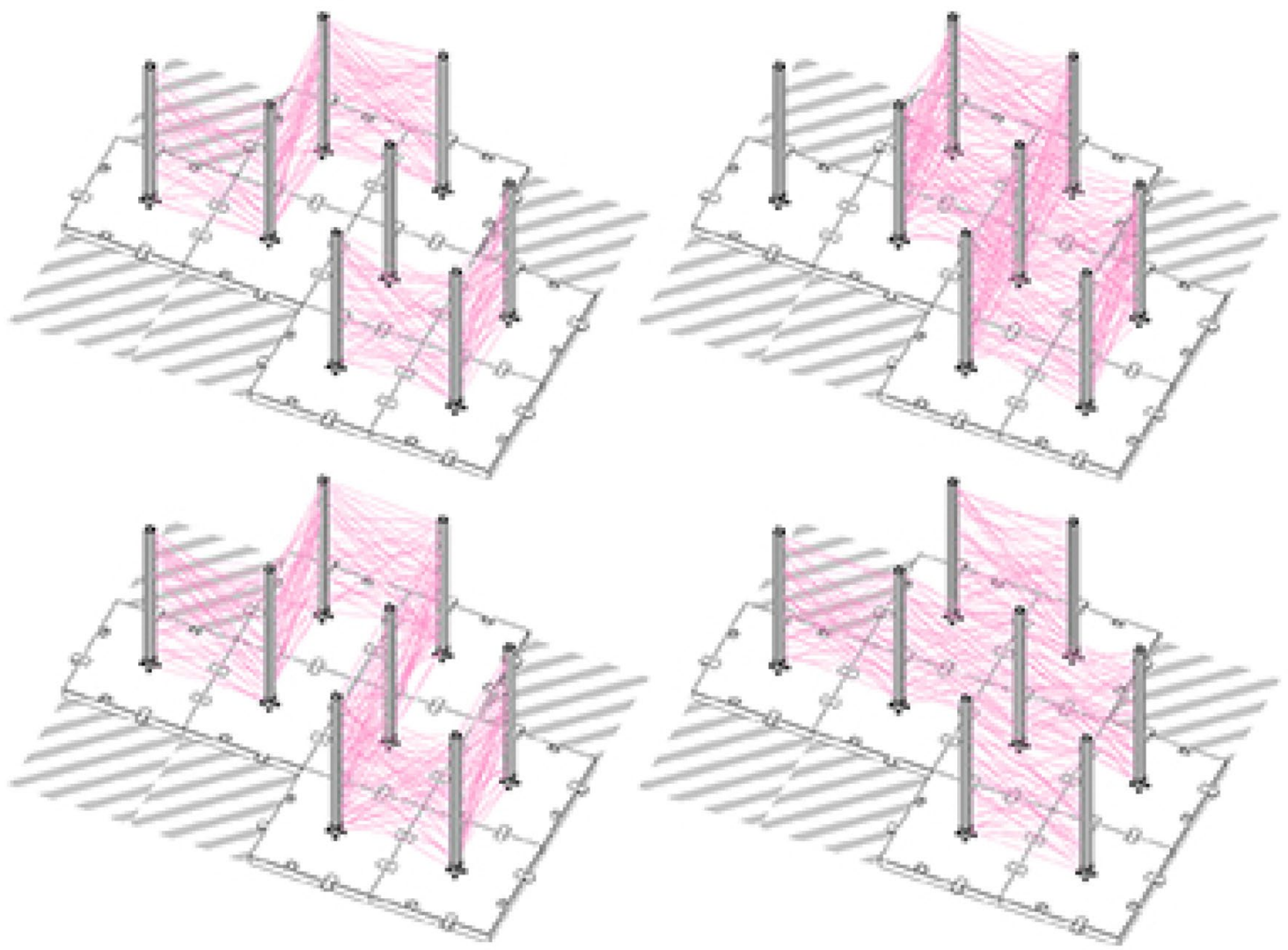

Fig. 7 Examples of design solutions within one post configuration

of surface disinfecting (World Health Organzation 2020) and airborne particle penetrability into the design evaluation criteria.

\subsection{Robotic system evaluation and future development}

In its current state of development, the robotic gantry takes approximately $30 \mathrm{~s}$ to wind a single filament strand (a combination of two anchoring routines and one travel routine). It is acknowledged that at a scale of a single winding operation, this speed is comparable if not slower than that of a human worker. However, during the manual partition winding it became clear that following the defined anchor sequence in a manual process requires additional time to identify the next anchor at every winding iteration, which renders the overall manual process to be significantly slower. Nonetheless manual winding allows to produce patterns that are not fabricable by the robotic system. For instance, when depositing a filament strand at a steep angle (more than $30^{\circ}$ ), the anchoring routine fails, caused by filament slippage around the anchor. In the manual process this limitation is not present, afforded by human dexterity. Further development of the winding effector is necessary to address this limitation. It is also recognized that the robots were wired to the control computer for data and wired to an outlet for power, and that future iterations into the robot hardware would introduce rail-integrated power supply and wireless network communication.

\subsection{Design tool and methodology evaluation and future development}

In its current stage, the global and local design tools are not integrated into a seamless design process. Thus, a feedback loop between the two is missing. Additionally, both local and global digital tools require further developments regarding the user interface. We have speculated that the interface allows for end users to effect spatial reconfiguration to meet their changing programmatic needs, but, at its current stage significant knowledge and programming skills are required to prototype and develop novel winding behaviors, making it inaccessible to a non-skilled user.

The evaluation criteria for the design proposals were defined by the workshop participants at the early stage of the development. They were aiming to create a variety of 
spaces based on the functional program. The diversity of design solutions generated (Fig. 7), including the ones based on the same post configuration, demonstrated the potential to differentiate spatial qualities through finely controlling the winding parameters.

Current evaluation of design options is relying almost exclusively on user intuition. Additional architectural evaluation criteria are to be developed, and a means of weighting these metrics for users with different priorities is to be integrated, either within the Computational Design Tool itself or as an external data set. An outcome of this evaluation criteria specificity could manifest itself in a catalog of ready-to-go spatial configurations and scenarios for known requirements (e.g., meeting, personal space, recreation area, etc.) allowing for a system that operates at two levels: pre-computed spatial solutions for typical users and an advanced mode that allows for tailoring solutions at a lower input level.

A future iteration of the software system would likely be implemented in a cloud server to allow for remote control of the system as a web host through a browser interface. Ideally, the core software logic would focus on modular layout automation with respect to the existing building structure and a generative module. This would maximize the end users' agency by allowing them to author custom compatible analysis modules to select optimal design space results relative to the criteria of their specific architectural program.

\section{Outlook}

The Soft Office project is addressing the issues of visual and programmatic zoning and reconfiguration within the context of interior commercial space. Moreover, it is contributing to the discussion of designing architecture as a temporal system, rather than a finalized object. It establishes a framework for further development of design and robotic approaches to adaptive architectural systems, specifically focusing on the relationship between the programmatic user input and the architectural and robotic outputs. Further research is necessary to identify methodologies for exercising user agency over complex design spaces where optimized solutions are executed semi-autonomously.

The prototyped workspace configuration system proposes an approach to reconfigurable architecture where the tasks are divided between the user and the machine based on the complexity of automation as well as temporal characteristics of change. Potentially, architectural elements proposed in this project can themselves become interaction devices, assuming a more intuitive way of communicating users' needs to the occupied space, thus opening a possibility to view space as an interface. This interface is operable not only by designers but by the architecture's occupants, allowing for real-time reconfiguration. This implies that architects increasingly design processes (Bier and Knight 2014), shifting the focus away from a static architectural artifact towards an operable time-based system. Ultimately, this means that an architectural product is beginning to operate on a time scale closer to that of other contemporary digital systems, potentially enabling a new degree of cultural and economic relevance of the architectural object.

\section{References}

Bailey C, Phelan N, Cosgrove A, Davis D (2018) This room is too dark and the shape is too long: quantifying architectural design to predict successful spaces. In: De Rycke K, Gengnagel C, Baverel O, Burry J, Mueller C, Nguyen MM, Rahm P, Thomsen MR (eds) Humanizing digital reality: design modelling symposium Paris 2017. Springer Singapore, Singapore, pp 337-348

Bier H (ed) (2018) Robotic building. Springer International Publishing, Cham

Bier H, Knight T (2014) Data-driven design to production and operation. FOOTPRINT. https://doi.org/10.7480/footprint.8.2.807

Bock T, Linner T (2015) Robot-oriented design: design and management tools for the deployment of automation and robotics in construction. Cambridge University Press, Cambridge

Bock T, Linner T (2016) Construction robots: elementary technologies and single-task construction robots. Cambridge University Press, Cambridge

Cheng L (2016) Towards embedding high-resolution intelligence into the built environment. Archidoct 4(1):29-40

Dierichs K, Menges A (2017) Granular construction: designed particles for macro-scale architectural structures. Archit Design 87(4):8893. https://doi.org/10.1002/ad.2200

Duffy F (2008) Lumbering to extinction in the digital field: the Taylorist office building. Harv Design Mag 29(2):2008-2009

Green KE (2016) Architectural robotics: ecosystems of bits, bytes, and biology. The MIT Press, Cambridge

Gross MD, Green KE (2012) Architectural robotics, inevitably. Interactions 19(1):28. https://doi.org/10.1145/2065327.2065335

Houayek H, Green KE, Gugerty L, Walker ID, Witte J (2014) AWE: an animated work environment for working with physical and digital tools and artifacts. Pers Ubiquit Comput 18(5):1227-1241. https ://doi.org/10.1007/s00779-013-0731-6

Kelly K (1994) Out of control: the new biology of machines, social systems and the economic world, vol 1. Addison-Wesley, Reading (paperback printing)

Kilian A (2018) The flexing room architectural robot: an actuated active bending robotic structure using human feedback. In: Anzalone P, Signore M, Wit AJ (eds) ACADIA 2018 Recalibration: on imprecision and infidelity. Proceedings of the 38th annual conference of the association for computer aided design in architecture. ACADIA publishing company

KUKA Roboter GmbH (2015) LBR iiwa 7 R800, LBR iiwa 14 R820 specification. KUKA Roboter GmbH, Augsburg

Lu J et al (2020) COVID-19 outbreak associated with air conditioning in Restaurant, Guangzhou, China, 2020. Emerg Infect Dis 26(7):1628-1631. https://doi.org/10.3201/eid2607.200764

Mitchell WJ (1999) E-topia: 'Urban life, Jim-but not as we know it.' MIT Press, Cambridge

Negroponte N (1975) Soft architecture machines. The MIT Press, Cambridge 
Park SY et al (2020) Coronavirus disease outbreak in Call Center, South Korea. Emerg Infect Dis 26(8):1666-1670. https://doi. org/10.3201/eid2608.201274

Sadler S (2005) Archigram: architecture without architecture. The MIT Press, Cambridge

Schafer GJ, Fullerton SK, Walker I, Vijaykumar A, Green KE (2018) Words Become Worlds: the LIT ROOM, a literacy support tool at room-scale. In: Proceedings of the 2018 on designing interactive systems conference 2018-DIS'18, Hong Kong, China, pp 511-522. https://doi.org/10.1145/3196709.3196728.

Sparrman B et al (2017) Large-scale lightweight transformable structures. In: Proceedings of the 37th annual conference of the association for computer aided design in architecture, Cambridge, MA, 2017, pp 572-581

Steenson MW (2017) Architectural intelligence: how designers and architects created the digital landscape. The MIT Press, Cambridge

Stumm S, Brell-Çokcan S (2019) Haptic programming. In: Willmann J, Block P, Hutter M, Byrne K, Schork T (eds) Robotic fabrication in architecture, art and design 2018. Springer International Publishing, Cham, pp 44-58

Turner PV (1987) La Formation de Le Corbusier: idéalisme et mouvement moderne. Ed. Macula, Coëtlogon
Wood D, Yablonina M, Aflalo M, Chen J, Tahanzadeh B, Menges A (2019) Cyber physical macro material as a UAV [re]configurable architectural system. In: Willmann J, Block P, Hutter M, Byrne K, Schork T (eds) Robotic fabrication in architecture, art and design 2018. Springer International Publishing, Cham, pp 320-335

World Health Organization (2020) Getting your workplace ready for COVID-19: how COVID-19 spreads. World Health Organization [Online]. Available: https://www.who.int/publications/m/item/ getting-your-workplace-ready-for-covid-19-how-covid-19-spreads

Yablonina M, Menges A (2019) Distributed fabrication: cooperative making with larger groups of smaller machines. Archit Design 89(2):62-69. https://doi.org/10.1002/ad.2413

Yablonina M, Nicolas KK, Menges A (2021) Designing [with] machines: task- and site-specific robotic teams for in-situ architectural making. In: Presented at the ACADIA 2020, 2021

Publisher's Note Springer Nature remains neutral with regard to jurisdictional claims in published maps and institutional affiliations. 